\title{
Charge symmetry violation in the parton distributions of the nucleon
}

\author{
Fu-Guang Cao* and A. I. Signal[ \\ Institute of Fundamental Sciences \\ Massey University \\ Private Bag 11 222, Palmerston North \\ New Zealand
}

\begin{abstract}
We point out that charge symmetry violation in both the valence and sea quark distributions of the nucleon has a non-perturbative source. We calculate this nonperturbative charge symmetry violation using the meson cloud model, which has earlier been successfully applied to both the study of $\mathrm{SU}(2)$ flavour asymmetry in the nucleon sea and quark-antiquark asymmetry in the nucleon. We find that the charge symmetry violation in the valence quark distribution is well below $1 \%$, which is consistent with most low energy tests but significantly smaller than the quark model prediction about $5 \%-10 \%$. Our prediction for the charge symmetry violation in the sea quark distribution is also much smaller than the quark model calculation.
\end{abstract}

PACS numbers: 12.39.-x; 11.30.Er

Keywords: Charge symmetry, Meson cloud, Parton distribution

*E-mail address: f.g.cao@massey.ac.nz.

†E-mail address: a.i.signal@massey.ac.nz. 


\section{INTRODUCTION}

Recently charge symmetry violation (CSV) in the parton distributions of the nucleon has attracted great interest. It has been generally believed that charge symmetry (CS) was highly respected in the nucleon system. Most low energy experiments have shown that CS is satisfied within about $1 \%$ in reaction amplitudes [1], and most high energy tests are also consistent with the charge symmetry, although generally with less precision than the low energy tests (for a recent review see [2] and references therein). However some theoretical calculations have suggested that the CSV in the valence quark distributions may be as large as $5 \%-10 \%$ 3 8 which is rather large compared with the low-energy results. There have been proposed a number of experiments in which CSV may be observed [9].

A serious challenge to CS has come from the comparison of the $F_{2}$ structure functions measured in charged and uncharged lepton deep inelastic scattering [11,10] performed by Boros, Londergan and Thomas [12]. A significantly larger CSV than the expectations of both theory [3 8] and other experiments [13] was found for the $s$ and $\bar{s}$ distributions in the low- $x$ region $(x<0.1)$. Any unexpected large CSV will greatly affect our understanding of non-perturbative dynamics and hadronic structure [2], and also the extraction of $\sin ^{2} \theta_{W}$ from neutrino scattering [3]. More recently, Boros, Steffens, Londergan and Thomas [14] performed a similar analysis to Ref. [12] with improved corrections for nuclear shadowing and the charm threshold in the neutrino data. They found that the data (including the low$x$ region where a large discrepancy was found in [12]) are consistent with charge symmetry within experimental errors and the present uncertainty in the strange quark distribution of the nucleon.

Thus suggestions of any large CSV in the parton distributions of the nucleon result from theoretical calculations [3 8 . Most of these theoretical calculations are performed using a quark model, such as the MIT bag model [4], Los Alamos Potential Model [5, 6], or a model independent version [3],7]. The quark model calculations are based on a quark-spectator (quark-diquark) picture of the nucleon in deep inelastic reactions, which is questionable in 
the low- $x$ region. Hence the quark model predictions for the low- $x$ behaviour of CSV are not very reliable. For the sea quark content, quark model calculations involve spectator states containing four quarks, which is also unreliable in the low- $x$ region as the mass parameter for these four-quark (or three-quark one-antiquark) states is less well-determined than that for the diquark states. CSV has also been estimated using the light-cone baryon-meson fluctuation model [8]. However, this calculation is mostly qualitative - the quantitative calculations are highly dependant on the model parameters. More theoretical study on CSV from a different point of view than the quark model will be worthwhile.

In this paper, we point out that the CSV in both the valence quark and sea quark has the same non-perturbative source, and that the meson cloud model (MCM) - which has been successfully applied to the study of the sea quark content of nucleon (including SU(2)

flavour asymmetry [15, 19], and $s$ and $\bar{s}$ content of the nucleon [16,20,21]) - can provide a natural explanation of CSV in the valence quark and sea quark distributions of the nucleon. We shall make an alternative investigation of CSV in the parton distributions of the nucleon by using the meson cloud model instead of the more commonly used quark model. Our calculations for the CSV are significantly different from the quark model predictions.

\section{CHARGE SYMMETRY VIOLATION IN THE MESON CLOUD MODEL}

Charge symmetry results from the $180^{\circ}$-rotation invariance of the strong Hamiltonian about the 2-axis in isospace [2]. At the quark level charge symmetry implies the invariance of a system under the interchange of up and down quarks. For the valence and sea parton distributions, this results in the following relations:

$$
\begin{array}{ll}
u_{v}^{p}(x)=d_{v}^{n}(x), & d_{v}^{p}(x)=u_{v}^{n}(x), \\
\bar{u}^{p}(x)=\bar{d}^{n}(x), & \bar{d}^{p}(x)=\bar{u}^{n}(x), \\
s^{p}(x)=s^{n}(x), & \bar{s}^{p}(x)=\bar{s}^{n}(x) .
\end{array}
$$

The charge symmetry violation in the parton distributions of the nucleon can be 'measured' via the quantities: 


$$
\begin{array}{cc}
\delta d_{v}(x)=d_{v}^{p}(x)-u_{v}^{n}(x), & \delta u_{v}(x)=u_{v}^{p}(x)-d_{v}^{n}(x), \\
\delta \bar{d}(x)=\bar{d}^{p}(x)-\bar{u}^{n}(x), & \delta \bar{u}(x)=\bar{u}^{p}(x)-\bar{d}^{n}(x), \\
\delta s(x)=s^{p}(x)-s^{n}(x), & \delta \bar{s}(x)=\bar{s}^{p}(x)-\bar{s}^{n}(x) .
\end{array}
$$

Before entering detailed calculation, it is helpful to break down the parton distribution in the nucleon into three parts: the parton distribution in the bare nucleon, the perturbative contribution, and the non-perturbative contribution i.e.

$$
\begin{gathered}
d^{p}=d_{\mathrm{bare}}^{p}+d_{\mathrm{per}}^{p}+d_{\mathrm{non}}^{p}, \quad u^{p}=u_{\mathrm{bare}}^{p}+u_{\mathrm{per}}^{p}+u_{\mathrm{non}}^{p} \\
\bar{d}^{p}=\bar{d}_{\mathrm{per}}^{p}+\bar{d}_{\mathrm{non}}^{p}, \quad \bar{u}^{p}=\bar{u}_{\mathrm{per}}^{p}+\bar{u}_{\mathrm{non}}^{p} \\
s^{p}=s_{\mathrm{per}}^{p}+s_{\mathrm{non}}^{p}, \quad \bar{s}^{p}=\bar{s}_{\mathrm{per}}^{p}+\bar{s}_{\mathrm{non}}^{p}
\end{gathered}
$$

Similar relations exist for the parton distribution of the neutron. We expect that the bare part obeys the charge symmetry

$$
d_{\mathrm{bare}}^{p}=u_{\mathrm{bare}}^{n}, \quad u_{\mathrm{bare}}^{p}=d_{\mathrm{bare}}^{n}
$$

The perturbative sea is produced in a very short time via gluon splitting, thus we expect the perturbative sea to also be $\mathrm{SU}(2)$ flavour symmetric,

$$
\bar{d}_{\mathrm{per}}^{p}=\bar{u}_{\mathrm{per}}^{p}, \quad \bar{u}_{\mathrm{per}}^{p}=\bar{d}_{\mathrm{per}}^{n}
$$

quark-antiquark symmetric,

$$
q_{\mathrm{per}}^{p, n}=\bar{q}_{\mathrm{per}}^{p, n}, \quad(q=u, d, s)
$$

and charge symmetric,

$$
\begin{array}{ll}
d_{\mathrm{per}}^{p}=u_{\mathrm{per}}^{n}, & u_{\mathrm{per}}^{p}=d_{\mathrm{per}}^{n}, \\
\bar{d}_{\mathrm{per}}^{p}=\bar{u}_{\mathrm{per}}^{n}, & \bar{u}_{\mathrm{per}}^{p}=\bar{d}_{\mathrm{per}}^{n}, \\
s_{\mathrm{per}}^{p}=s_{\mathrm{per}}^{n}, & \bar{s}_{\mathrm{per}}^{p}=\bar{s}_{\mathrm{per}}^{n} .
\end{array}
$$

From the bare parton distribution being charge symmetric [Eq. (10)] and the perturbative sea being quark-antiquark symmetric [Eq. (12)] and charge symmetric [Eqs. (13) and (14)] we have the CS violating valence distributions 


$$
\begin{aligned}
& \delta d_{v}=\left(d_{\text {non }}^{p}-\bar{d}_{\text {non }}^{p}\right)-\left(u_{\text {non }}^{n}-\bar{u}_{\text {non }}^{n}\right), \\
& \delta u_{v}=\left(u_{\text {non }}^{p}-\bar{u}_{\text {non }}^{p}\right)-\left(d_{\text {non }}^{n}-\bar{d}_{\text {non }}^{n}\right) .
\end{aligned}
$$

Using the charge symmetry of the perturbative sea [Eqs. (13)-(15)] we can obtain the CS violating sea distributions

$$
\begin{gathered}
\delta \bar{d}=\bar{d}_{\mathrm{non}}^{p}-\bar{u}_{\mathrm{non}}^{n}, \quad \delta \bar{u}=\bar{u}_{\mathrm{non}}^{p}-\bar{d}_{\mathrm{non}}^{n}, \\
\delta s=s_{\mathrm{non}}^{p}-s_{\mathrm{non}}^{n}, \quad \delta \bar{s}=\bar{s}_{\mathrm{non}}^{p}-\bar{s}_{\mathrm{non}}^{n} .
\end{gathered}
$$

Thus the charge symmetry violation in both the valence and the sea distributions has a non-perturbative origin.

The meson cloud model (MCM) is a model of the non-perturbative contribution to the quark distributions of the nucleon. It can provide natural explanations of the flavour asymmetry in the nucleon sea [15, 19] and quark-antiquark asymmetry [16,20,21] in the nucleon. The essential point of the MCM is that the nucleon can fluctuate into different baryon-meson Fock states,

$$
|N\rangle_{\text {physical }}=Z|N\rangle_{\text {bare }}+\sum_{B M} \sum_{\lambda \lambda^{\prime}} \int d y d^{2} \mathbf{k}_{\perp} \phi_{B M}^{\lambda \lambda^{\prime}}\left(y, k_{\perp}^{2}\right)\left|B^{\lambda}\left(y, \mathbf{k}_{\perp}\right) ; M^{\lambda^{\prime}}\left(1-y,-\mathbf{k}_{\perp}\right)\right\rangle
$$

where $Z$ is the wave function renormalization constant, $\phi_{B M}^{\lambda \lambda^{\prime}}\left(y, k_{\perp}^{2}\right)$ is the wave function of the Fock state containing a baryon $(B)$ with longitudinal momentum fraction $y$, transverse momentum $\mathbf{k}_{\perp}$, and helicity $\lambda$, and a meson $(M)$ with momentum fraction $1-y$, transverse momentum $-\mathbf{k}_{\perp}$, and helicity $\lambda^{\prime}$. The model assumes that the lifetime of a virtual baryonmeson Fock state is much larger than the interaction time in the deep inelastic or Drell-Yan process, thus the contribution from the virtual baryon-meson Fock states to the quark and anti-quark distributions of the nucleon can be written as convolutions

$$
\begin{aligned}
& q_{\text {non }}(x)=\sum_{B M}\left[\int_{x}^{1} \frac{d y}{y} f_{B M / N}(y) q^{B}\left(\frac{x}{y}\right)+\int_{x}^{1} \frac{d y}{y} f_{M B / N}(1-y) q^{M}\left(\frac{x}{y}\right)\right], \\
& \bar{q}_{\text {non }}(x)=\sum_{B M} \int_{x}^{1} \frac{d y}{y} f_{M B / N}(1-y) \bar{q}^{M}\left(\frac{x}{y}\right),
\end{aligned}
$$

where $f_{B M / N}(y)=f_{M B / N}(1-y)$ is fluctuation function which gives the probability for the nucleon fluctuating into a virtual $B M$ state 


$$
f_{B M / N}(y)=\int_{0}^{\infty} d k_{\perp}^{2}\left|\phi_{B M}\left(y, k_{\perp}^{2}\right)\right|^{2}
$$

As the proton and neutron form an $\mathrm{SU}(2)$ isospin doublet, the baryons and mesons in their respective virtual Fock states differ only in their carried charge. If we neglect the mass differences among these baryons and mesons the fluctuation functions for the proton and neutron will be the same. Thus the contributions to the parton distribution of the nucleon from these fluctuations will be isospin symmetric (charge symmetric). As is well known, the electromagnetic interaction induces mass differences among these baryons and mesons. If we take into account these mass differences, the probabilities for the corresponding fluctuations of proton and neutron will be different and thus the contributions to the parton distributions of the proton and neutron will be different, which results in CSV in the parton distributions of the nucleon. Thus the MCM can provide a natural explanation of CSV in the parton distributions of the nucleon. Although it is argued from the quark model calculations that the electromagnetic effect does not play a significant role in the calculation of CSV in the parton distributions, it is worthwhile to study this effect using a different theoretical picture.

For the CSV in the up and down quark distributions, we consider the fluctuations $N \rightarrow$ $N \pi$ and $N \rightarrow \Delta \pi$, but neglect the other fluctuations such as $N \rightarrow N(\Delta) \rho, N \rightarrow N \eta(\omega)$ and $N \rightarrow \Delta \eta(\omega)$ due to the higher masses of the involved mesons. Thus the fluctuations we consider include:

$$
\begin{aligned}
p(u u d) \rightarrow n(u d d)+\pi^{+}(u \bar{d}), & n(u d d) \rightarrow p(u u d)+\pi^{-}(\bar{u} d), \\
p(u u d) \rightarrow \Delta^{0}(u d d)+\pi^{+}(u \bar{d}), & n(u d d) \rightarrow \Delta^{+}(u u d)+\pi^{-}(\bar{u} d), \\
p(u u d) \rightarrow p(u u d)+\pi^{0}([u \bar{u}+d \bar{d}] / \sqrt{2}), & n(u d d) \rightarrow n(u d d)+\pi^{0}([u \bar{u}+d \bar{d}] / \sqrt{2}), \\
p(u u d) \rightarrow \Delta^{+}(u u d)+\pi^{0}([u \bar{u}+d \bar{d}] / \sqrt{2}), & n(u d d) \rightarrow \Delta^{0}(u d d)+\pi^{0}([u \bar{u}+d \bar{d}] / \sqrt{2}), \\
p(u u d) \rightarrow \Delta^{++}(u u u)+\pi^{-}(\bar{u} d), & n(u d d) \rightarrow \Delta^{-}(d d d)+\pi^{+}(u \bar{d}) .
\end{aligned}
$$

For the CSV in the strange and anti-strange quark distributions we consider the following fluctuations

$$
p(u u d) \rightarrow \Lambda(u d s)+K^{+}(u \bar{s}), \quad n(u d d) \rightarrow \Lambda(u d s)+K^{0}(d \bar{s})
$$




$$
\begin{aligned}
& p(u u d) \rightarrow \Sigma^{0}(u d s)+K^{+}(u \bar{s}), \quad n(u d d) \rightarrow \Sigma^{0}(u d s)+K^{0}(d \bar{s}), \\
& p(u u d) \rightarrow \Sigma^{+}(u u s)+K^{0}(d \bar{s}), \quad n(u d d) \rightarrow \Sigma^{-}(d d s)+K^{+}(u \bar{s}) .
\end{aligned}
$$

Since we consider the CSV between the proton and neutron, we should not neglect the mass difference between the proton and neutron, $m_{p}-m_{n}=-1.3 \mathrm{MeV}$, and those among the baryon and meson multiplets [22],

$$
\begin{aligned}
& m_{\Delta^{-}}-m_{\Delta^{0}}=m_{\Delta^{0}}-m_{\Delta^{+}}=m_{\Delta^{+}}-m_{\Delta^{++}}=1.3 \mathrm{MeV} \\
& m_{\Sigma^{-}}-m_{\Sigma^{0}}=4.8 \mathrm{MeV} \\
& m_{\Sigma^{0}}-m_{\Sigma^{+}}=3.3 \mathrm{MeV} \\
& m_{\pi^{ \pm}}-m_{\pi^{0}}=4.6 \mathrm{MeV} \\
& m_{K^{0}}-m_{K^{+}}=4.0 \mathrm{MeV}
\end{aligned}
$$

The probabilities of various fluctuations can be calculated using the effective Lagrangian and time-ordered perturbation theory in the infinite momentum frame. For the fluctuations $N \rightarrow N \pi, N \rightarrow \Lambda K$ and $N \rightarrow \Sigma K$, the fluctuation functions can be expressed as 16,18

$$
f_{B M / N}(y)=C_{1} \frac{g_{N B M}^{2}}{16 \pi^{2}} \int_{0}^{\infty} \frac{d k_{\perp}^{2}}{y(1-y)} \frac{G_{B M}^{2}\left(M_{B M}^{2}\right)}{\left(m_{N}^{2}-m_{B M}^{2}\right)^{2}} \frac{k_{\perp}^{2}+\left(y m_{N}-m_{B}\right)^{2}}{y}
$$

where $y$ is the longitudinal momentum fraction of the baryon $B, g_{N B M}$ is the effective coupling constant, $m_{B M}^{2}$ is the invariant mass squared of the $B M$ Fock state,

$$
m_{B M}^{2}=\frac{m_{B}^{2}+k_{\perp}^{2}}{y}+\frac{m_{\pi}^{2}+k_{\perp}^{2}}{1-y}
$$

and $G_{B M}$ is the phenomenological form factor, for which we adopt the exponential form

$$
G_{B M}\left(y, k_{\perp}^{2}\right)=\exp \left[\frac{m_{N}^{2}-m_{B M}^{2}\left(y, k_{\perp}^{2}\right)}{2 \Lambda^{2}}\right]
$$

$\Lambda$ is a cut-off parameter which can be taken as $\Lambda=1.08 \mathrm{GeV}$ for all fluctuations involving octet baryons and pseudoscalar or vector mesons [16]. The effective coupling constants are taken to be $g_{N N \pi}=13.07$ [16,18], $g_{N \Lambda K}=13.12$ and $g_{N \Sigma K}=6.82$ [20,23. The coefficient $C_{1}$ in Eq. (27) comes from the Clebsch-Gordan coefficients for the fluctuations of different 
isospin multiplets. $C_{1}=1$ for $f_{p \pi^{0} / p}, f_{n \pi^{0} / n}, f_{\Lambda K^{+} / p}, f_{\Lambda K^{0} / n}, f_{\Sigma^{0} K^{+} / p}$ and $f_{\Sigma^{-} K^{0} / n}$, and $C_{1}=2$ for $f_{n \pi^{+} / p}, f_{p \pi^{-} / n}, f_{\Sigma^{+} K^{0} / p}$, and $f_{\Sigma^{-} K^{+} / n}$. For the fluctuation $N \rightarrow \Delta \pi$ we have [16, 18]

$f_{\Delta \pi / N}(y)=C_{2} \frac{g^{2}}{16 \pi^{2}} \int_{0}^{\infty} \frac{d k_{\perp}^{2}}{y(1-y)} \frac{G_{\Delta \pi}^{2}\left(M_{\Delta \pi}^{2}\right)}{\left(m_{N}^{2}-m_{\Delta \pi}^{2}\right)^{2}} \frac{\left[k_{\perp}^{2}+\left(m_{\Delta}-y m_{N}\right)^{2}\right]\left[k_{\perp}^{2}+\left(m_{\Delta}+y m_{N}\right)^{2}\right]^{2}}{6 m_{\Delta}^{2} y^{3}}$,

where $g=11.8 \mathrm{GeV}^{-1}$ [16],18], $C_{2}=1$ for $f_{\Delta^{++} \pi^{-} / p}$ and $f_{\Delta^{-} \pi^{+} / n}, C_{2}=2 / 3$ for $f_{\Delta^{+} \pi^{0} / p}$ and $f_{\Delta^{0} \pi^{0} / n}$, and $C_{2}=1 / 3$ for $f_{\Delta^{0} \pi^{+} / p}$ and $f_{\Delta^{+} \pi^{-} / p}$. We adopt the exponential form [Eq. (29)] for the form factor and the cut-off parameter was taken to be $\Lambda=0.98 \mathrm{GeV}$ 18].

In the meson cloud model the non-perturbative contribution to the quark and the antiquark distributions in the nucleon sea come from the quarks and anti-quarks of the baryons $(N, \Lambda, \Sigma)$ and mesons $(\pi, K)$ in the virtual baryon-meson Fock states. So we need the parton distributions in the involved baryons and mesons as input. For the parton distribution in the pion, we employ the parameterization given by Glück, Reya, and Stratmann (GRS98) [24] and we neglect the sea content in the meson, that is,

$$
\begin{aligned}
\bar{d}^{\pi^{+}}=u^{\pi^{+}} & =\bar{u}^{\pi^{-}}=d^{\pi^{-}}=\frac{1}{2} v^{\pi}, \\
\bar{u}^{\pi^{0}}=u^{\pi^{0}} & =\bar{d}^{\pi^{0}}=d^{\pi^{0}}=\frac{1}{4} v^{\pi}, \\
v^{\pi}\left(x, \mu_{\mathrm{NLO}}^{2}\right) & =1.052 x^{-0.495}(1+0.357 \sqrt{x})(1-x)^{0.365},
\end{aligned}
$$

at scale $\mu_{\mathrm{NLO}}^{2}=0.34 \mathrm{GeV}^{2}$. For the $\bar{s}$ distribution in the $K^{+}$and $K^{0}$ we use the GRS98 parameterization [24]

$$
\bar{s}^{K^{+}}\left(x, \mu_{\mathrm{NLO}}^{2}\right)=\bar{s}^{K^{0}}\left(x, \mu_{\mathrm{NLO}}^{2}\right)=\left[1-0.540(1-x)^{0.17}\right] v^{\pi}\left(x, \mu_{\mathrm{NLO}}^{2}\right)
$$

at scale $\mu_{\mathrm{NLO}}^{2}=0.34 \mathrm{GeV}^{2}$. For the quark distributions in the bare baryons, we first use the up and down quark distributions in the proton given by Glück, Reya, and Vogt (GRV98) [25],

$$
\begin{aligned}
& d^{p}\left(x, \mu_{\mathrm{NLO}}^{2}\right)=0.624(1-x) u^{p}\left(x, \mu_{\mathrm{NLO}}^{2}\right) \\
& u^{p}\left(x, \mu_{\mathrm{NLO}}^{2}\right)=0.632 x^{-0.57}(1-x)^{3.09}(1+18.2 x),
\end{aligned}
$$


at scale $\mu_{\mathrm{NLO}}^{2}=0.40 \mathrm{GeV}^{2}$, then relate these to the distributions in the other baryons via the relations

$$
\begin{gathered}
d^{n}=u^{\Delta^{+}}=d^{\Delta^{0}}=u^{p}, \quad u^{n}=d^{\Delta^{+}}=u^{\Delta^{0}}=d^{p}, \\
u^{\Delta^{++}}=u^{p}+d^{p}, \quad d^{\Delta^{-}}=u^{p}+d^{p}, \\
s^{\Lambda}=s^{\Sigma^{+}}=s^{\Sigma^{0}}=s^{\Sigma^{-}}=\frac{1}{2} u^{p} .
\end{gathered}
$$

We evolve the distributions to the scale $Q^{2}=4 \mathrm{GeV}^{2}$ using the program of Miyama and Kumano [26] in which the evolution equation is solved numerically using a brute-force method. We found that at $Q^{2}=4 \mathrm{GeV}^{2}$ the parton distributions we need $\left(v^{\pi}\left(x, Q^{2}\right), \bar{s}^{0}\left(x, Q^{2}\right)\right.$ $u^{p}\left(x, Q^{2}\right)$ and $\left.d^{p}\left(x, Q^{2}\right)\right)$ can be parametrized using the following form

$$
q\left(x, Q^{2}\right)=a x^{b}(1-x)^{c}(1+d \sqrt{x}+e x)
$$

with the parameters given in Table 1 . We estimate the uncertainty in solving the evolution equations numerically and parametrizating the parton distribution in the form of Eq. (40) to be about $2 \%$ in the $x$-region which we are interested in ie $x>10^{-3}$.

The final expressions for the CSV in the valence parton distributions are given by:

$$
\begin{aligned}
x \delta d_{v}=\int_{0}^{x} d y & \frac{x}{y}\left\{\left[f_{n \pi^{+} / p}(y)-f_{p \pi^{-} / n}(y)\right] u^{p}\left(\frac{x}{y}\right)\right. \\
- & {\left[f_{n \pi^{+} / p}(1-y)-f_{p \pi^{-} / n}(1-y)\right] \frac{1}{2} v^{\pi}\left(\frac{x}{y}\right) } \\
+ & {\left[f_{p \pi^{0} / p}(y)-f_{n \pi^{0} / n}(y)\right] d^{p}\left(\frac{x}{y}\right) } \\
+ & {\left[f_{\Delta^{0} \pi^{+} / p}(y)-f_{\Delta^{+} \pi^{-} / n}(y)\right] u^{p}\left(\frac{x}{y}\right) } \\
- & {\left[f_{\Delta^{0} \pi^{+} / p}(1-y)-f_{\Delta^{+} \pi^{-} / n}(1-y)\right] \frac{1}{2} v^{\pi}\left(\frac{x}{y}\right) } \\
+ & {\left[f_{\Delta^{+} \pi^{0} / p}(y)-f_{\Delta^{0} \pi^{0} / n}(y)\right] d^{p}\left(\frac{x}{y}\right) } \\
+ & {\left.\left[f_{\Delta^{++} \pi^{-} / p}(1-y)-f_{\Delta^{-} \pi^{+} / n}(1-y)\right] \frac{1}{2} v^{\pi}\left(\frac{x}{y}\right)\right\}, } \\
x \delta u_{v}=\int_{0}^{x} d y & \frac{x}{y}\left\{\left[f_{n \pi^{+} / p}(y)-f_{p \pi^{-} / n}(y)\right] d^{p}\left(\frac{x}{y}\right)\right. \\
- & {\left[f_{n \pi^{+} / p}(1-y)-f_{p \pi^{-} / n}(1-y)\right] \frac{1}{2} v^{\pi}\left(\frac{x}{y}\right) }
\end{aligned}
$$




$$
\begin{aligned}
& +\left[f_{p \pi^{0} / p}(y)-f_{n \pi^{0} / n}(y)\right] u^{p}\left(\frac{x}{y}\right) \\
& +\left[f_{\Delta^{0} \pi^{+} / p}(y)-f_{\Delta^{+} \pi^{-} / n}(y)\right] d^{p}\left(\frac{x}{y}\right) \\
& -\left[f_{\Delta^{0} \pi^{+} / p}(1-y)-f_{\Delta^{+} \pi^{-} / n}(1-y)\right] \frac{1}{2} v^{\pi}\left(\frac{x}{y}\right) \\
& +\left[f_{\Delta^{+} \pi^{0} / p}(y)-f_{\Delta^{0} \pi^{0} / n}(y)\right] u^{p}\left(\frac{x}{y}\right) \\
& +\left[f_{\Delta^{++} \pi^{-} / p}(y)-f_{\Delta^{-} \pi^{+} / n}(y)\right]\left(u^{p}+d^{p}\right) \\
& \left.+\left[f_{\Delta^{++} \pi^{-} / p}(1-y)-f_{\Delta^{-} \pi^{+} / n}(1-y)\right] \frac{1}{2} v^{\pi}\left(\frac{x}{y}\right)\right\} .
\end{aligned}
$$

For CSV in the sea we obtain

$$
\begin{aligned}
& x \delta \bar{d}=\int_{0}^{x} d y \frac{x}{y}\left\{f_{n \pi^{+} / p}(1-y)-f_{p \pi^{-} / n}(1-y)+\frac{1}{2}\left[f_{p \pi^{0} / p}(1-y)-f_{n \pi^{0} / n}(1-y)\right]\right. \\
& +f_{\Delta^{0} \pi^{+} / p}(1-y)-f_{\Delta^{+} \pi^{-} / n}(1-y) \\
& \left.+\frac{1}{2}\left[f_{\Delta^{+} \pi^{0} / p}(1-y)-f_{\Delta^{0} \pi^{0} / n}(1-y)\right]\right\} \frac{1}{2} v^{\pi}\left(\frac{x}{y}\right) \\
& x \delta \bar{u}=\int_{0}^{x} d y \frac{x}{y}\left\{\frac{1}{2}\left[f_{p \pi^{0} / p}(1-y)-f_{n \pi^{0} / n}(1-y)\right]+\frac{1}{2}\left[f_{\Delta^{+} \pi^{0} / p}(1-y)-f_{\Delta^{0} \pi^{0} / n}(1-y)\right]\right. \\
& \left.+f_{\Delta^{++} \pi^{-} / p}(1-y)-f_{\Delta^{-} \pi^{+} / n}(1-y)\right\} \frac{1}{2} v^{\pi}\left(\frac{x}{y}\right), \\
& x \delta s=\int_{0}^{x} d y \frac{x}{y}\left\{f_{\Lambda K^{+} / p}(y)-f_{\Lambda K^{0} / n}(y)+f_{\Sigma^{0} K^{+} / p}(y)-f_{\Sigma^{0} K^{0} / n}(y)\right. \\
& \left.+f_{\Sigma^{+} K^{0} / p}(y)-f_{\Sigma^{-} K^{+} / n}(y)\right\} \frac{1}{2} u^{p}\left(\frac{x}{y}\right) \\
& x \delta \bar{s}=\int_{0}^{x} d y \frac{x}{y}\left\{f_{\Lambda K^{+} / p}(1-y)-f_{\Lambda K^{0} / n}(1-y)+f_{\Sigma^{0} K^{+} / p}(1-y)-f_{\Sigma^{0} K^{0} / n}(1-y)\right. \\
& \left.+f_{\Sigma^{+} K^{0} / p}(1-y)-f_{\Sigma^{-} K^{+} / n}(1-y)\right\} \frac{1}{2} \bar{s}^{K}\left(\frac{x}{y}\right)
\end{aligned}
$$

\section{RESULT AND DISCUSSION}

From Eqs. (41) - (46) we can see explicitly that the differences among various fluctuation

functions such as $f_{n \pi^{+} / p}$ and $f_{p \pi^{-} / n}$ result in the CSV in the parton distributions of the nucleon. We plot these differences in Figs. 1-3. It can be seen that the difference in the 
fluctuation $N \rightarrow N \pi\left(f_{n \pi^{+} / p}-f_{p \pi^{-} / n}, \ldots\right)$ is much larger than that in the fluctuation $N \rightarrow \Delta \pi\left(f_{\Delta^{++} \pi^{-} / p}-f_{\Delta^{+} \pi^{+} / n}, \ldots\right)$ and the latter is much bigger than that in the fluctuation $N \rightarrow \Lambda K\left(f_{\Lambda \pi^{+} / p}-f_{\Lambda K^{0} / n}, \ldots\right)$. Thus the CSV in the valence and sea up and down quarks should be the same order, and both larger than the CSV in the $s$ and $\bar{s}$ distributions. The difference $f_{n \pi^{+} / p}-f_{p \pi^{-} / n}$ is much larger than $f_{p \pi^{0} / p}-f_{n \pi^{0} / n}$, thus the CSV in the sea of the minority quark flavor $(\delta \bar{d})$ will be much larger than that of the majority quark flavor $(\delta \bar{u})$ due to the absence of $\left(f_{n \pi^{+} / p}-f_{p \pi^{-} / n}\right)$ term in $\delta \bar{u}$. The probabilities of the various fluctuations can be obtained by integrating the corresponding fluctuation functions. We find the probabilities of the dominant fluctuations to be

$$
\begin{gathered}
P\left(p \rightarrow n \pi^{+}\right)=0.202 \quad P\left(n \rightarrow p \pi^{-}\right)=0.205 \\
P\left(p \rightarrow \Delta^{++} \pi^{-}\right)=0.0481 \quad P\left(n \rightarrow \Delta^{-} \pi^{+}\right)=0.0475 \\
P\left(p \rightarrow \Lambda K^{+}\right)=0.0127 \quad P\left(n \rightarrow \Lambda K^{0}\right)=0.0125
\end{gathered}
$$

that is there is about a $1 \%$ excess of fluctuations $n \rightarrow p \pi^{-}$over $p \rightarrow n \pi^{+}$and $p \rightarrow \Delta^{++} \pi^{-}$ over $n \rightarrow \Delta^{-} \pi^{+}$, and about $2 \%$ excess of $p \rightarrow \Lambda K^{+}$over $n \rightarrow \Lambda K^{0}$.

We present our results for the CSV in the valence quark sector $\left(x \delta d_{v}\right.$ and $\left.x \delta u_{v}\right)$ in Fig. 4. We find that $x \delta d_{v}$ and $x \delta u_{v}$ have similar shape and both are negative, which is quite different from the quark model prediction of $x \delta d_{v}$ being positive for most values of $x$ [3 7]. Furthermore, our numerical results are about $10 \%$ of the quark model estimation [3] [7]. It has been argued that although the absolute values of $\delta d_{v}$ and $\delta u_{v}$ are small, the ratio $R_{\text {min }}=\delta d_{v} / d_{v}^{p}$ may be much larger than the ratio $R_{\text {maj }}=\delta u_{v} / u_{v}^{p}$ in the large- $x$ region since the $d_{v}^{p}(x) / u_{v}^{p}(x) \ll 1 / 2$ as $x \rightarrow 0$, and values as large as $5 \% \sim 10 \%$ [3] have been obtained for the ratio $\delta d_{v} / d_{v}^{p}$. No such large- $x$ enhancement appears in our calculation for both ratios. We find that the ratio $\delta d_{v} / d_{v}^{p}$ exhibits a maximum about $0.2 \%$ at $x=0.1$ while the ratio $\delta u_{v} / u_{v}^{p}$ diverges as $x \rightarrow 0$ but is smaller than $0.3 \%$ in the region of $x>0.02$. The numerical results for the CSV in the sea quark $(x \delta \bar{d}, x \delta \bar{u}, x \delta s$ and $x \delta \bar{s})$ are given Fig. 5. We find that $x \delta \bar{d}$ has the largest CSV and that $x \delta d_{v}$ and $x \delta u_{v}$ are of similar magnitude, which is consistent with our expectation from the analysis of the fluctuation functions. Our 
prediction for the $x \delta \bar{d}$ being negative is opposite to the positive theoretical prediction in [7].

Our calculation for the low- $x$ behaviours of $x \delta \bar{d}, x \delta \bar{u}, x \delta s$ and $x \delta \bar{s}$ are also quite different from the quark model prediction [0] - the quark model predicts that these quantities diverge as $x \rightarrow 0$ while our calculations show all these CSV distributions go to 0 as $x \rightarrow 0$. We did not find any significant large CSV in the sea quark distribution of the nucleon, which is consistent with the most recent phenomenological analysis [14]. We would like to emphasise that instead of the quark model we adopt a totally different model, the meson cloud model, to calculate the charge symmetry violation in the parton distributions of the nucleon. The quark model calculation in the small- $x$ region is not very reliable since the quark-diquark picture that is employed breaks down in this region. The meson cloud model is suitable in the study of the CSV in the parton distribution of the nucleon since it has the same non-perturbative origin as the $\bar{d} / \bar{u}$ asymmetry in the proton.

\section{CRITICISM OF QUARK MODEL CALCULATIONS}

We have already mentioned a few of the difficulties with CSV calculations using quark models. A recent paper by Benesh and Londergan [7] attempted to avoid any quark model specifics and relate possible CSV in the valence quark distributions to the measured valence distributions. Starting from the parton model expression for a quark distribution [27]

$$
q(x)=p^{+} \sum_{n} \delta\left(p^{+}(1-x)-p_{n}^{+}\right)|\langle n|\Psi(0)| p\rangle|^{2}
$$

where the intermediate state $|n\rangle$ has 4-momentum $p_{n}$ and the + components of momenta are defined by $k^{+}=k^{0}+k^{z}$, and then making the assumption that the intermediate state can be modelled by a diquark system with definite mass $M_{d}$, Benesh and Londergan investigate the consequences for CSV of varying $M_{d}$. Following the Adelaide group [28], we can attempt to determine the dependence of the quark distribution on $M_{d}$. Assuming that the modulus squared of the wavefunction for the struck quark in the nucleon is symmetric about the z-axis, we can use the delta function to perform the integration over transverse diquark momenta 


$$
\int d \mathbf{p}_{n} \delta\left(p^{+}(1-x)-p_{n}^{+}\right)=2 \pi \int_{p_{\min }}^{\infty} d p_{n} p_{n}
$$

where

$$
\begin{aligned}
p_{\text {min }} & =\left|\frac{M^{2}(1-x)^{2}-M_{d}^{2}}{2 M(1-x)}\right| \\
p_{T} & =2 M(1-x) \sqrt{M_{d}^{2}+\mathbf{p}_{n}^{2}}-M^{2}(1-x)^{2}-M_{d}^{2}
\end{aligned}
$$

and $M$ is the nucleon mass. Therefore we obtain the quark distribution in the form

$$
q(x)=\int_{p_{\min }\left(x, M_{d}\right)}^{\infty} d p_{n} g\left(p_{n}\right)
$$

where $g(k)$ only depends on the magnitude of the 3-momentum (this is not true in the case of spin dependent quark distributions), and we have reminded ourselves that $p_{\min }$ is a function of $x$ and $M_{d}$. Thus all the $M_{d}$ dependence of the quark distribution is in the lower limit of the integral. Use of the fundamental theorem of calculus then gives

$$
\begin{aligned}
\frac{\partial q(x)}{\partial M_{d}} & =\frac{\partial q(x)}{\partial x} \frac{\partial p_{\min }}{\partial M_{d}} / \frac{\partial p_{\min }}{\partial x} \\
& =\frac{2 M_{d}(1-x)}{M^{2}(1-x)^{2}+M_{d}^{2}} \frac{\partial q(x)}{\partial x} .
\end{aligned}
$$

This expression is similar to that of Benesh and Londergan [7], except that in their case the $\partial / \partial x$ operator acts on the product of the kinematic factor $2 M_{d}(1-x) /\left(M^{2}(1-x)^{2}+M_{d}^{2}\right)$ and the original quark distribution. The derivation of reference [7] differs from ours in that they make a variation in $M_{d}$ under the integral in equation (50), then evaluate the integral over $\mathbf{p}_{n}$ by ignoring any transverse momenta of the diquark. However in our expression, all transverse momenta have been properly integrated over (in the parton model the transverse momentum of the struck quark vanishes).

Benesh and Londergan then use the idea of Close and Thomas [29] that the quark model $S U(4)$ spin-isospin symmetry is broken by the color hyperfine interaction. The hyperfine interaction leads to a splitting in the masses of the spin-0 and spin-1 diquark states and hence to a difference between the up and down valence distributions:

$$
\begin{aligned}
& u_{v}(x)=\frac{3}{2} q_{v}^{s}(x)+\frac{1}{2} q_{v}^{t}(x) \\
& d_{v}(x)=q_{v}^{t}(x)
\end{aligned}
$$


where the superscripts $s, t$ refer to singlet and triplet diquark states respectively. If the $N-\Delta$ mass splitting is also caused by the color hyperfine interaction, then the shifts in the singlet and triplet diquark masses are found to be

$$
\delta_{h f} M_{d}^{t}=-\frac{1}{3} \delta_{h f} M_{d}^{s}=+50 \mathrm{MeV}
$$

By now expanding $q_{v}^{s}\left(x, M_{d}\right)$ and $q_{v}^{t}\left(x, M_{d}\right)$ in Taylor series to first order in $\delta M_{d}$ about the symmetry point $M_{d}=M_{d}^{0}, q_{v}^{s}\left(x, M_{d}^{0}\right)=q_{v}^{t}\left(x, M_{d}^{0}\right)$ Benesh and Londergan obtain the shift in the triplet quark distribution

$$
\delta_{h f} q_{v}^{t}(x)=\frac{1}{6}\left(2 d_{v}(x)-u_{v}(x)\right)
$$

Now as the CSV at the quark level comes from quark mass and electromagnetic effects, both of which are iso-vector, the only mass shift is in $M_{d}^{t}$, and to first order the shift in the triplet quark distribution will be proportional to that from the hyperfine interaction

$$
\delta_{C S V} q_{v}^{t}(x)=\frac{\delta_{C S V} M_{d}^{t}}{\delta_{h f} M_{d}^{t}} \frac{2 d_{v}(x)-u_{v}(x)}{6} .
$$

The main difficulty with this argument is that it is entirely based on first order shifts in the quark distributions. However the second order terms can be estimated, and they are of similar magnitude to the first order terms. Expanding the quark distributions to second order in $\delta M_{d}$ about the symmetry point we have

$q_{v}\left(x, M_{d}^{0}+\delta M_{d}\right)=q_{v}\left(x, M_{d}^{0}\right)+\left.\delta M_{d} \frac{\partial q_{v}\left(x, M_{d}\right)}{\partial M_{d}}\right|_{M_{d}=M_{d}^{0}}+\left.\frac{1}{2}\left(\delta M_{d}\right)^{2} \frac{\partial^{2} q_{v}\left(x, M_{d}\right)}{\partial\left(M_{d}\right)^{2}}\right|_{M_{d}=M_{d}^{0}}$

where the partial derivatives on the right hand side can be evaluated using (56). This then gives for the hyperfine shift in the triplet quark distribution

$$
\begin{aligned}
\delta_{h f} q_{v}^{t}(x) & =-\frac{1}{3} \delta_{h f} q_{v}^{s}(x)+\left.2\left(\delta M_{d}^{t}\right)^{2} \frac{\partial^{2} q_{v}\left(x, M_{d}\right)}{\partial\left(M_{d}\right)^{2}}\right|_{M_{d}=M_{d}^{0}} \\
& =\frac{1}{6}\left(2 d_{v}(x)-u_{v}(x)\right)+\left.\frac{3}{2}\left(\delta M_{d}^{t}\right)^{2} \frac{\partial^{2} q_{v}\left(x, M_{d}\right)}{\partial\left(M_{d}\right)^{2}}\right|_{M_{d}=M_{d}^{0}}
\end{aligned}
$$

As an estimate of the second order term we can approximate $q_{v}\left(x, M_{d}^{0}\right)$ by $d_{v} x$ or $\left(u_{v}(x)+\right.$ $\left.d_{v}(x)\right) / 3$ and use one of the well-known parametrizations of the quark valence distributions 
[25,30]. We also take our value for $M_{d}^{0}$ to lie in the range $(0.65-0.85) M$, which is the range suggested by quark models, though the results are not very sensitive to the value of $M_{d}^{0}$ in this range. In Fig. 6 we compare the first and second order terms for $\delta_{h f} q_{v}^{t}(x)$. We can see that our estimate of the second order term is of comparable magnitude to the first order term over most of the $x$-range. Indeed for low $x$ it is larger, showing the unreliability of quark model calculations in this region. For $x>0.2$ the second order term is of opposite sign to the first order term, which indicates that the first order estimate of the shift in the quark distribution is too large in this region. This in turn implies that the estimate of CSV induced shifts in the quark distributions in this region are also too large. These conclusions are not much influenced by the choice of valence quark parametrization, the value chosen for $M_{d}^{0}$, or whether we use Benesh and Londergan's expression for the dependence of the quark distribution on $M_{d}$ rather than equation (56). The reason for these conclusions not being greatly influenced by the choice of expression for $\partial q(x) / \partial M_{d}$ is that, with the quark distributions used, the highest order derivative term in $x$ always dominates. This is a consequence of the divergences in the valence quark distributions near $x=0, q_{v}(x) \sim x^{-0.5}$ in all cases.

\section{SUMMARY}

Although it has been generally assumed that charge symmetry was highly respected in the nucleon system, there have been some phenomenological analysis [12,14] and theoretical calculations [3] 8] about the possible extent of CSV in the parton distributions. Any unexpected large CSV will greatly affect our understanding on the non-perturbative dynamics and hadronic structure, and the extraction of $\sin ^{2} \theta_{W}$ from neutrino scattering. Up to now most theoretical attempts to calculate the CSV in the parton distributions are based on the quark model and employ the quark-diquark model. In this paper we point out that CSV in both

the valence and sea quark distributions of the nucleon can arise from the non-perturbative dynamics of the nucleon. We present an alternative analysis of CSV in the parton distribu- 
tions employing the meson cloud model, which has previously been successful in the study of the flavour asymmetry and the quark-antiquark asymmetry of the nucleon. In the meson cloud model the proton and neutron may fluctuate into hadron-meson Fock states in which the hadrons and mesons are in different charged states respectively. As we consider the mass differences among these hadrons and mesons, the probabilities of proton and neutron fluctuating into the corresponding Fock states will be different. Thus the non-perturbative contributions to the valence and sea quarks distributions will be different, which naturally leads to the CSV in both the valence and sea distributions of the nucleon. Our predictions for the CSV in the valence sector and sea sector are both different from the quark model calculations. We also point out the deficiencies of quark model based calculations of CSV in the parton distributions. In particular the quark-diquark picture is inadequate at low- $x$, and in the medium- $x$ region the use of a first order shift in the parton distributions must be questioned, as higher order shifts are of similar magnitude. The coming experimental information on the parton distributions of the nucleon and more theoretical studies on this issue will examine these calculations.

\section{ACKNOWLEDGMENTS}

This work was partially supported by the Massey Postdoctoral Foundation, New Zealand. We are grateful to Prof. A. W. Thomas for useful discussions. F. G. Cao would like to thank the Special Research Center for the Subatomic Structure of Matter for its hospitality. 


\section{REFERENCES}

[1] G. A. Miller, B. M. K. Nefkens, and I. Salus, Phys. Rep. 194 (1990) 1.

[2] J. T. Londergan and A. W. Thomas, in Progress in Particle and Nuclear Physics, Volume 41, P. 49, ed. A. Faessler (Elsevier Science, Amsterdam, 1998).

[3] E. Sather, Phys. Lett. B 274 (1992) 433.

[4] E. Rodionov, A. W. Thomas, and J. T. Londergan, Mod. Phys. Lett. A 9 (1994) 1799.

[5] C. J. Benesh and T. Goldman, Phys. Rev. C 55 (1997) 441.

[6] C. J. Benesh, T. Goldman, and G. J. Stephenson Jr., QCD 161, 1995, w3.

[7] C. J. Benesh and J. T. Londergan, Phys. Rev. C 58 (1998) 1218.

[8] B. Q. Ma, Phys. Lett. B 274 (1992) 115; Phys. Lett. B 408 (1997) 387; B. Q. Ma, A. Schäfer, and W. Creiner, Phys. Rev. D 47 (1993) 51.

[9] J. T. Londergan, G. T. Garvey, G. Q. Liu, E. N. Rodionov, and A. W. Thomas, Phys. Lett. B 340 (1994) 115; J. T. Londergan, A. Pang, and A. W. Thomas, Phys. Rev. D 54 (1996) 3154; J. T. Londergan, S. Braendler, and A. W. Thomas, Phys. Lett. B 424 (1998) 185; J. T. Londergan, Nucl. Phys. A629 (1998) 457c.

[10] W. G. Seligman et al., CCFR-Collaboration, Phys. Rev. Lett. 79 (1997) 1213; A. O. Bazarko et al., CCFR-Collaboration, Z. Phys. C 65 (1995) 189; S. A. Rabinowitz et al., CCFR-Collaboration, Phys. Rev. Lett. 70 (1993) 134.

[11] M. Arneodo et al., NMC-Collaboration, Nucl. Phys. B483 (1997) 3.

[12] C. Boros, J. T. Londergan, and A. W. Thomas, Phys. Rev. Lett. 81 (1998) 4075; Phys. Rev. D 59 (1999) 074021.

[13] F. Abe et al., CDF Collaboration, Phys. Rev. Lett. 81 (1998) 5744.

[14] C. Boros, F. M. Steffens, J. T. Londergan, and A. W. Thomas, hep-ph/9908280. 
[15] A. W. Thomas, Phys. Lett. B 126 (1983) 97.

[16] H. Holtmann, A. Szczurek, and J. Speth, Nucl. Phys. A 569 (1996) 631.

[17] J. Speth and A. W. Thomos, Adv. Nucl. Phys. 24 (1998) 83.

[18] W. Melnitchouk, J. Speth, and A. W. Thomas, Phys. Rev. D 59 (1998) 014033

[19] S. Kumano, Phys. Rep. 303 (1998) 103.

[20] A. I. Signal and A. W. Thomas, Phys. Lett. B 191 (1987) 205.

[21] F. G. Cao and A. I. Signal, Phys. Rev. D 60 (1999) 074021.

[22] Particle Data Group, C. Caso et al., Eur. Phys. J. C 3 (1998) 1.

[23] J. J. Aubert et. al., Phys. Lett. 123B (1983) 275

[24] M. Glück, E. Reya, and M. Stratmann, Eur. Phys. J. C 2 (1998) 159.

[25] M. Glück, E. Reya, and A. Vogt, Eur. Phys. J. C 5 (1998) 461.

[26] M. Miyama and S. Kumano, Comput. Phys. Commun. 94 (1996) 185.

[27] R. L. Jaffe, in Relativistic Dynamics and Quark-Nuclear Physics, edited by M. B. Johnson and A. Pickleseimer (Wiley, New York, 1985).

[28] A. W. Schreiber, A. I. Signal and A. W. Thomas, Phys. Rev. D 44 (1991) 2653.

[29] F. Close and A. W. Thomas Phys. Lett. 212B (1988) 227.

[30] A. D. Martin, R. G. Roberts, W. J. Stirling and R. S. Thorne, EPJC 4 (1998) 463; H. L. Lai et al., PRD 55 (1997) 1280. 
Table 1. Parameters in Eq. (40) at $Q^{2}=4 \mathrm{GeV}^{2}$.

\begin{tabular}{|c|c|c|c|c|c|}
\hline & $a$ & $b$ & $c$ & $d$ & $e$ \\
\hline$v^{\pi}\left(x, Q^{2}\right)$ & 1.712 & -0.518 & 1.182 & -0.836 & 0.972 \\
\hline $\bar{s}^{K}\left(x, Q^{2}\right)$ & 0.803 & -0.516 & 1.306 & -0.762 & 0.957 \\
\hline$u^{p}\left(x, Q^{2}\right)$ & 1.029 & -0.572 & 3.933 & 1.550 & 6.033 \\
\hline$d^{p}\left(x, Q^{2}\right)$ & 0.615 & -0.575 & 5.096 & 1.102 & 6.773 \\
\hline
\end{tabular}




\section{FIGURE CAPTIONS}

Fig. 1. The differences between fluctuation functions $f_{N \pi / p}$ and $f_{N \pi / n}$.

Fig. 2. The differences between fluctuation functions $f_{\Delta \pi / p}$ and $f_{\Delta \pi / n}$.

Fig. 3. The differences between fluctuation functions $f_{\Lambda(\Sigma) K / p}$ and $f_{\Lambda(\Sigma) K / n}$.

Fig. 4 The charge symmetry violation in the valence quark sector. $R_{\min }=\delta d_{v} / d_{v}^{p}$ and $R_{\mathrm{maj}}=\delta u_{v} / u_{v}^{p}$

Fig. 5 The charge symmetry violation in the sea quark sector.

Fig. 6 The first and second order shifts in the triplet quark distribution caused by the color hyperfine interaction. The solid curve is the first order shift $\left(2 d_{v}(x)-u_{v}(x)\right) / 6$ calculated using the parametrizations of reference [25]. The dashed curve is the second order shift estimated using $q(x)=\left(d_{v}(x)+u_{v}(x)\right) / 3, M_{d}^{0}=0.75 M$, and a mass shift of $50 \mathrm{MeV}$ for the triplet diquark state. 
Fig. 1

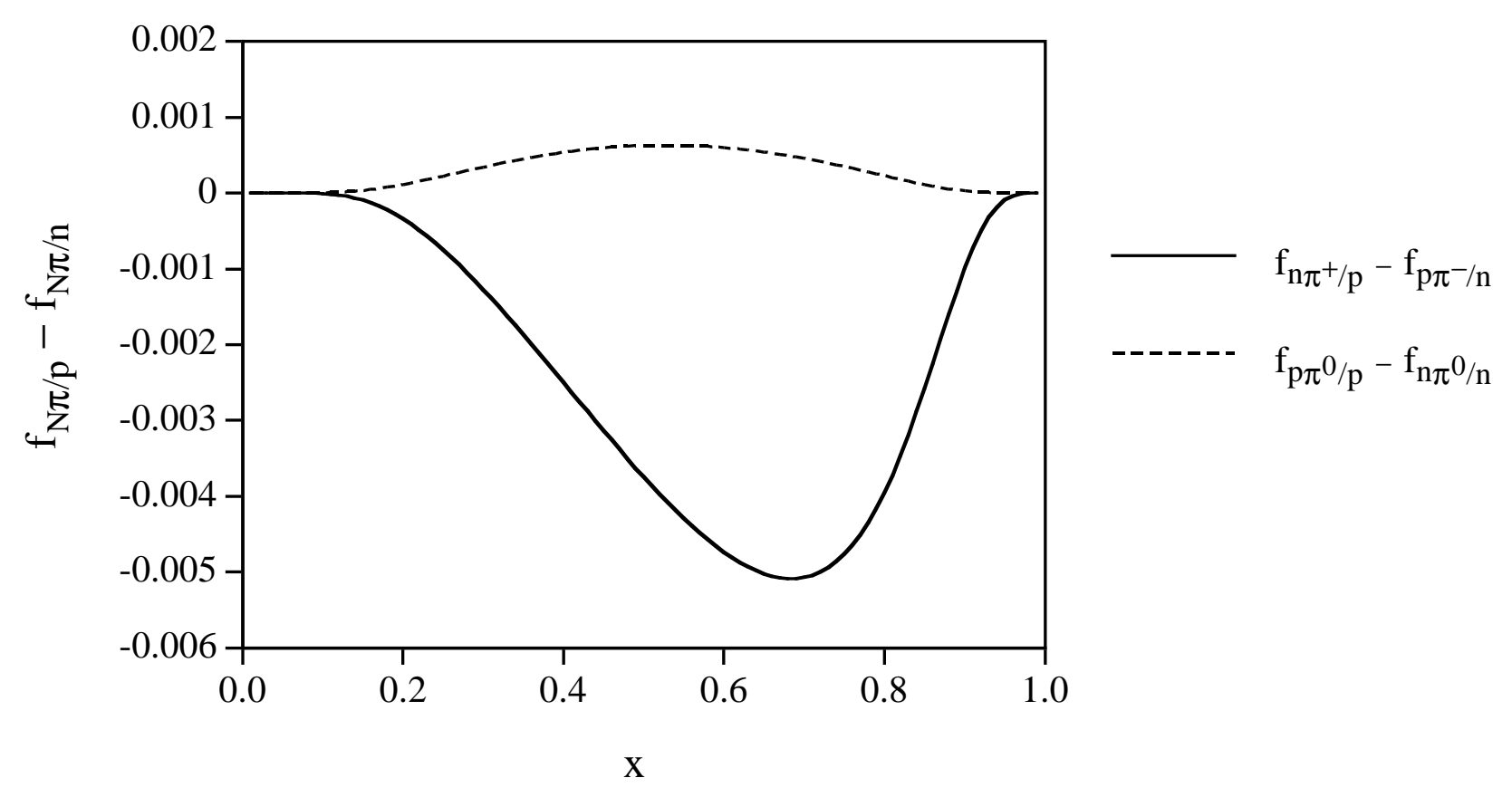


Fig. 2

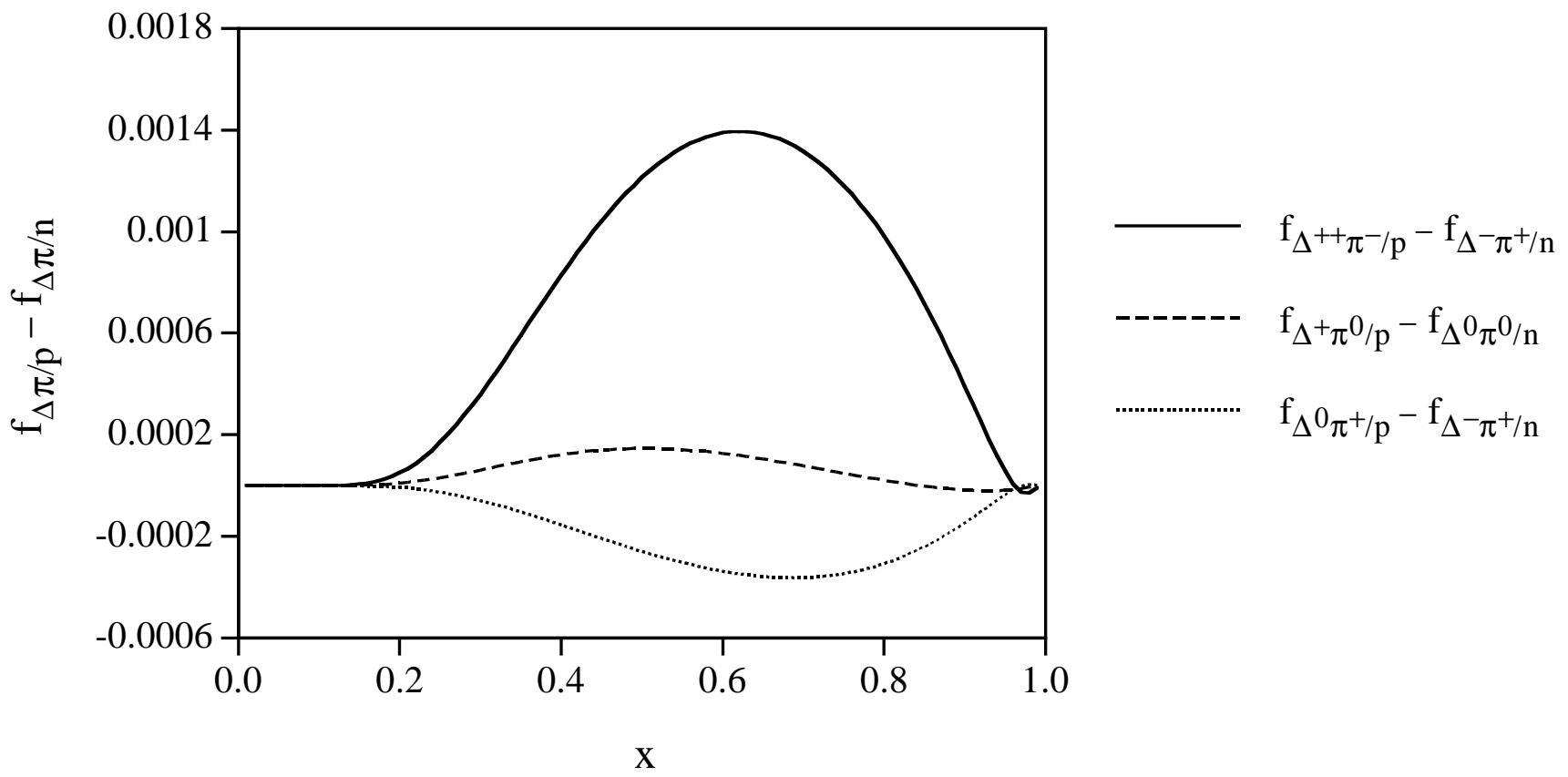


Fig. 3

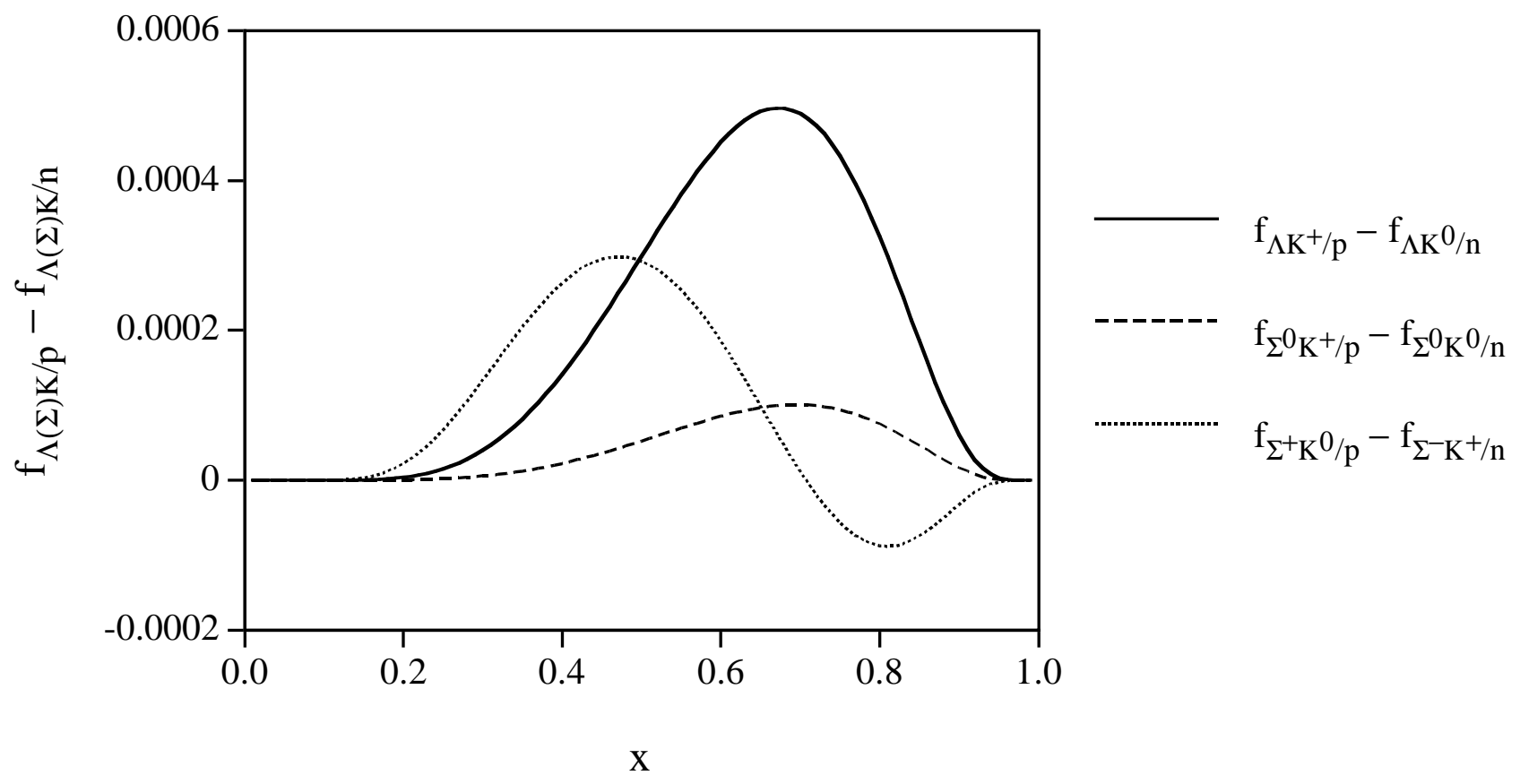


Fig. 4

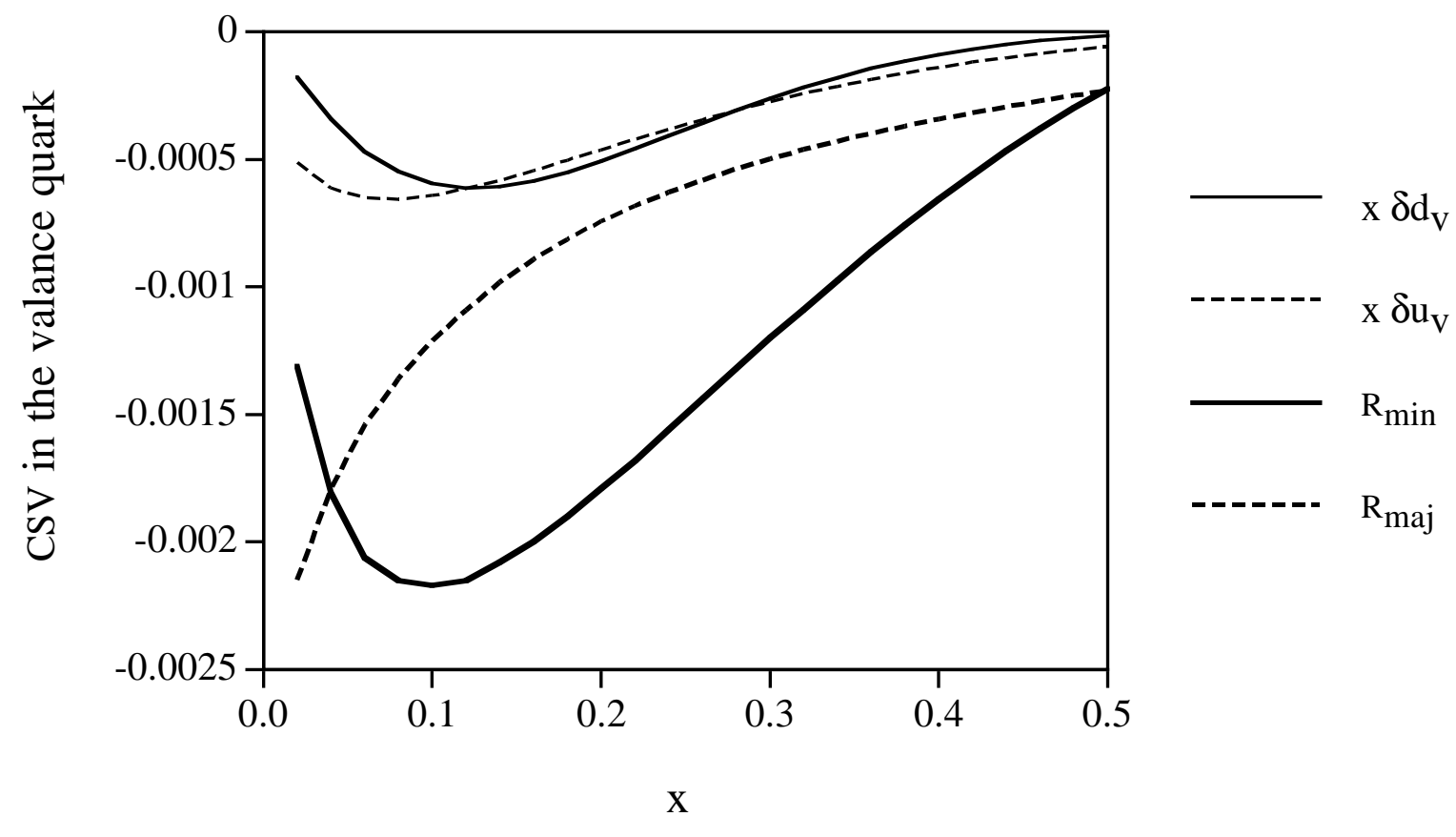


Fig. 5

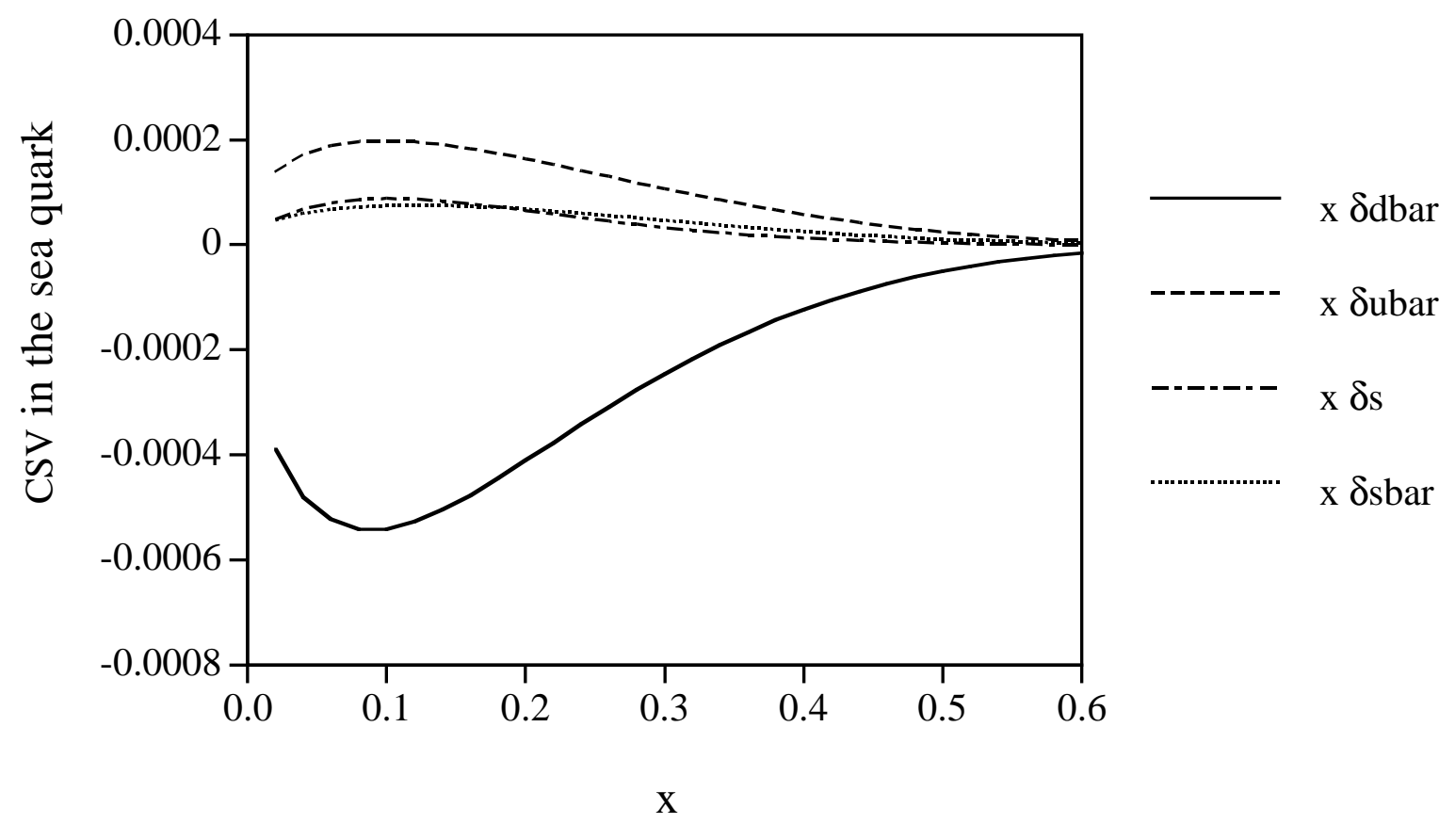


Fig. 6

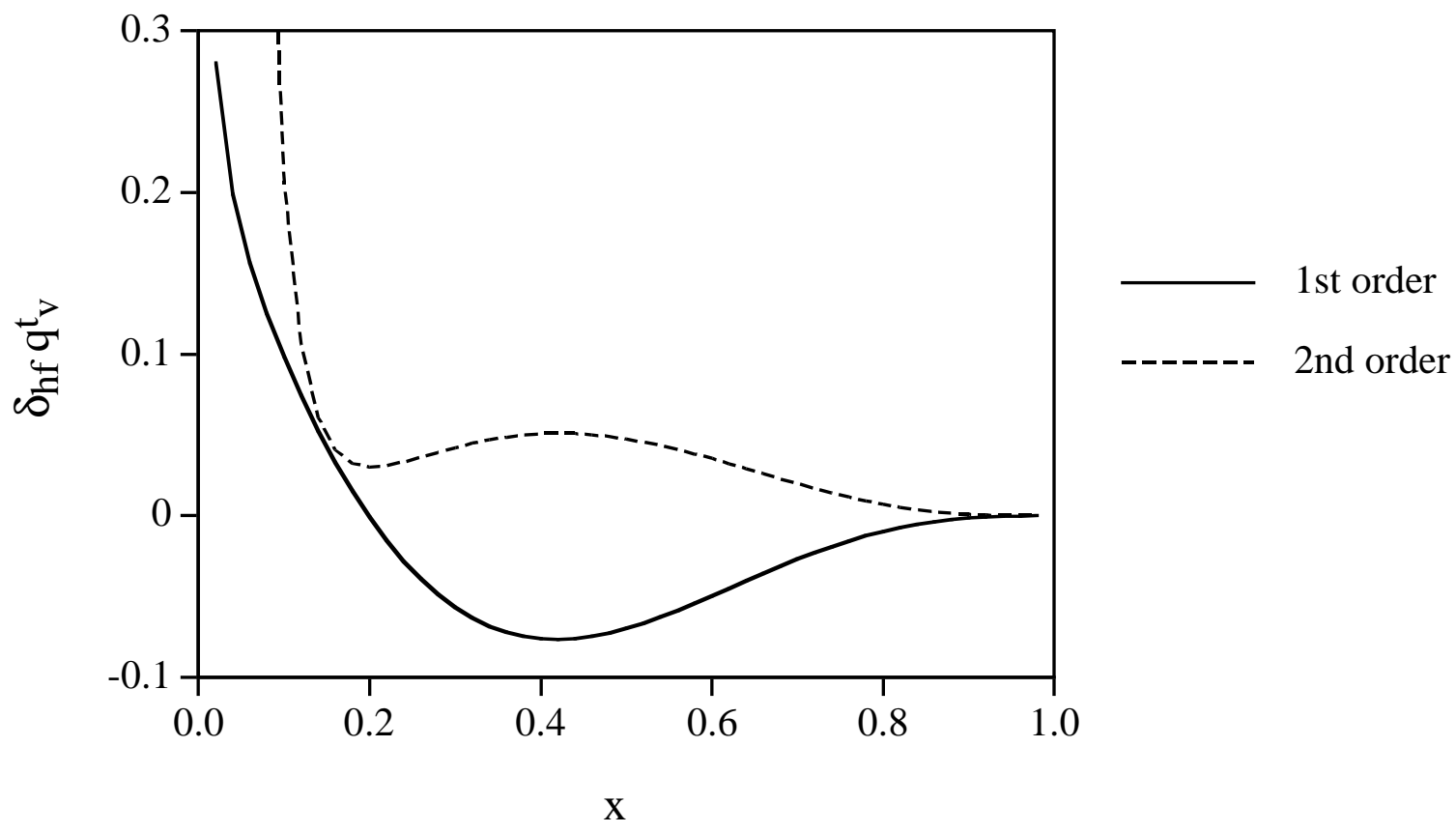

